\title{
Integrative physiology: facts and theories
}

\author{
Pietro E. di Prampero
}

Published online: 4 November 2008

(C) Springer-Verlag 2008

Nine years ago, upon accepting Dr. Rolf Lange's invitation, on behalf of Springer-Verlag, to become Editor-in-Chief of the European Journal of Applied Physiology, I was pondering the role of integrative and system physiology in the vast landscape of the life sciences. In spite of the scornful definition of "paleophysiology" by some younger colleagues, I was rather optimistic. Indeed, I stressed the desire of the Editors and Editorial Board of the journal to move towards a unifying view of physiology as a whole, from the subcellular and cellular levels to organs, systems, up to the conscious man (di Prampero 1999). This grand design is far from complete; however, when considering the number and quality of the articles dealing with this integrated approach published over these nine years, I am convinced that we are steadily moving in the right direction. So, I would like to thank our Authors, Editors, Editorial Board, Referees and above all our Readers for having supported my optimism with experimental evidence. I am also convinced that this trend will continue under the leadership of the new Editorin-Chief, Professor Susan Ward and Reviews Editor, Professor Dag Linnarsson. To them both I would like to express my personal esteem and friendship, as well as my gratitude for having accepted the "honor and onus" of steering the journal. I would also like to express my warmest thanks to my daughter Maria: without her generous, intelligent and efficient help, these nine years would have been much less pleasant and productive, and to the whole group of competent and efficient co-workers at Springer.

P. E. di Prampero $(\varangle)$

Dipartimento di Scienze e tecnologie biomediche,

Università degli Studi di Udine,

Piazzale M. Kolbe 4, 33100 Udine, Italy

e-mail: pprampero@mail.dstb.uniud.it
I will conclude these few paragraphs reporting some scientific speculations that I would like to throw in the scientific arena and that may show why I do think that integrative physiology is extraordinary and unique among the life sciences.

In the early seventies, I had the fortune of spending a memorable evening with Hermann Rahn and Rodolfo Margaria. The discussion among the three of us ranged from the mechanisms controlling energy metabolism at rest or during exercise, to the energy cost of running in different animals, to the relationships of the above with the animals' mass, to the meaning of biological time.

The essentials of this discussion, integrated by the information gained in the intervening thirty five years are summarised below.

The resting metabolic rate $\left(E_{\mathrm{r}}{ }^{\prime}\right)$ and the life span $(T)$ of animals are a function of their mass $(M)$, as described by: $E_{\mathrm{r}}=a \times M^{\sim 0.75}$ and $T=b \times M^{\sim 0.25}$. The constants $a$ and $b$ are different among the different classes of the animal kingdom (e.g., for $E_{\mathrm{r}}$ in kcal per day and $T$ in years: $a=69$ and $129 ; b=7.5$ and 21.6, for mammals and birds) (see Wilkie 1977; Rahn and Ar 1980; McMahon and Bonner 1983). However, irrespective of the values of $a$ and $b$, the sum of the two exponents is close to 1.0 , for all investigated classes. Therefore, the total amount of energy $\left(E_{\text {tot }}\right)$ spent by a given animal throughout his entire life cycle, as given by the product of $E_{\mathrm{r}} \times T$, is a linear function of his body mass: $E_{\mathrm{tot}}=E_{\mathrm{r}}{ }^{\prime} \times T=a \times b \times M^{\sim 1.0}$. As a consequence, the overall energy spent per unit body mass is constant and equal to: $E_{\text {tot }} \times M^{-1}=a \times b$. For mammals and birds $E_{\text {tot }} \times M^{-1}$ amounts to $1.9 \times 10^{5}$ and $1.1 \times 10^{6} \mathrm{kcal} \mathrm{kg}^{-1}$, respectively.

The fascinating regularities described above suggest that the resting metabolism, the "biological time" and the mass of a given animal are an entangled web, wherein the mass 
dictates the resting metabolism and this last the "biological time" of the animal. They are, however, rather difficult to interpret univocally. Indeed: (a) the mass of a given animal changes from juvenile to adult states, in some species increasing throughout the whole life cycle; (b) the life span is difficult to assess precisely for animals in the wild; (c) the metabolic rate $\left(E_{\mathrm{r}}{ }^{\prime}\right)$ applies strictly to the resting metabolism, as such it does not include the energy expenditure of locomotion and of other activities; (d) the applicability of allometric rules has been questioned on several grounds (Darveau et al. 2002; Weibel 2002).

However, also in avian eggs, in which the limitations reported above under (a)-(c) do not apply, and in which case $T$ can be viewed as the etching time, the amount of energy consumed by one unit of egg's mass from deposition to etching is constant: it amounts to about $500 \mathrm{kcal} \mathrm{kg}^{-1}\left(2.2 \mathrm{~kJ} \mathrm{~g}^{-1}\right)$ i.e. to about $0.05 \%$ of the overall amount of energy consumed by the bird in its subsequent life (Rahn and Ar 1980).

The temptation to consider the above regularity as a "law of nature" is difficult to resist. A possible interpretation follows. From deposition to etching, the fertilised egg is transformed from a single cell into a functional assembly of differentiated cells (the chick). In the course of this process, the information content of the system is increased, because of the synthesis of larger and more ordered structures from smaller and less ordered precursors. This process is rendered thermodynamically possible by the concomitant flow of energy through the system. The cost of information can be assumed to be $\geq k \ln 2 \mathrm{bit}^{-1}$, where $k$ is the Boltzmann constant $\left(1.38 \times 10^{-23} \mathrm{~J} \mathrm{~K}^{-1}\right)$, i.e. about $0.3 \times 10^{-23} \mathrm{~kJ}$ $\mathrm{bit}^{-1}$ at $310 \mathrm{~K}$ (Morowitz 1967). Therefore, since the overall energy flow is $2.2 \mathrm{~kJ} \mathrm{~g}^{-1}$, the information content of the egg, from deposition to etching, increases by $2.2 \mathrm{~kJ} \mathrm{~g}^{-1} \times\left(0.3 \times 10^{-23} \mathrm{~kJ} \mathrm{bit}^{-1}\right)^{-1}$, i.e. by $7.73 \times 10^{23}$ bits $\mathrm{g}^{-1}$. This is about one order of magnitude smaller than the information content of bacterial cells, which, according to Lehninger (1971), for E. coli "may greatly exceed" $10^{24}$ bit $\mathrm{g}^{-1}$.

It should be noted that: (1) the thermodynamic efficiency of the synthesis of large structures from smaller precursor, and (2) the initial information content of the avian egg, have been neglected. Indeed, both these approximations are trivial in view of the other assumptions and simplifications of the above estimates. However, the fact that the orders of magnitude of the information content as calculated from the statistical or the energetic approach are not too far apart is rather gratifying indeed.

Even in the light of the above "dangerously attractive" speculations, the reasons why a similar amount of energy (information) is consumed (gained) in a time which is shorter the smaller the egg remains elusive.

Returning now from eggs to animals and humans, it can be concluded that the biological time is somehow dictated by the rate of the animal's energy consumption, a consideration supported also by the observation by Boddington (1978) that the perceptual time depends on the metabolic rate of the brain. However, the underlying mechanism as well as the meaning of "biological time" keep escaping our understanding.

In concluding, I would like to dedicate these somewhat "wild" speculations to the memory of two "grand old masters" of integrative physiology, Rodolfo Margaria and Hermann Rahn, who for many years have been friends and teachers of many of us.

\section{References}

Boddington MJ (1978) An absolute metabolic scope for activity. J Theor Biol 75:443-449. doi:10.1016/0022-5193(78)90355-7

Darveau CA, Suarez RK, Andrews RD, Hochachka PW (2002) Allometric cascade as a unifying principle of body mass effects on metabolism. Nature 417:166-170. doi:10.1038/417166a

di Prampero PE (1999) Editorial. Eur J Appl Physiol 79:119. doi: $10.1007 /$ s004210050484

Lehninger AL (1971) Bioenergetics. WA Benjamin Inc, Menlo Park, pp 177-182

McMahon TA, Bonner JT (1983) On size and life scientific American library. WH Freeman and Company, New York, pp 111-225

Morowitz HJ (1967) Entropy for biologists. An introduction to thermodynamics. Academic Press, New York, pp 105-112

Rahn H, Ar A (1980) Gas exchange of the avian egg: time structure and function. Am Zool 20:477-484

Weibel ER (2002) The pitfalls of power laws. Nature 417:131-132. doi:10.1038/417131a

Wilkie DR (1977) Metabolism and body size. In: Pedley TJ (ed) Scale effects in animal locomotion. Academic Press, London, pp 22-36 